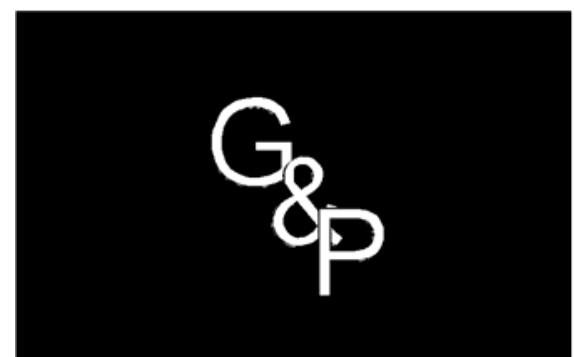

\title{
AVALIAÇÃO DE SISTEMAS DE MEDIÇÃO UTILIZANDO QUADRADOS LATINOS
}

\section{GESTÃO}

\&

\section{PRODUÇÃO}

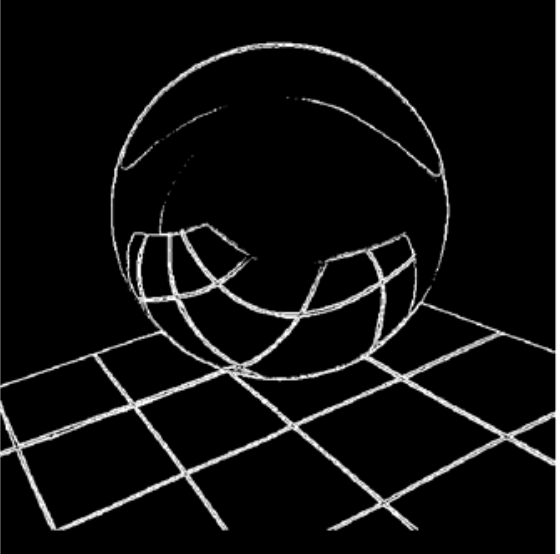

v.2, n.1 ,p.70-86, abr.1995
José Roberto do Rego

Engenheiro de Produção - Escola Politécnica da USP

Autolatina Operações de Caminhões e Ônibus Av. Henry Ford, 1787 - São Paulo - SP

F: (011) - $915-2414$

\section{Pedro Luiz de Oliveira Costa Neto}

Professor Doutor do

Departamento de Engenharia de Produção Escola Politécnica da Universidade de São Paulo

\section{Resumo}

São apresentados os métodos tradicionais utilizados para a avaliação de sistemas de medição. Esses métodos tradicionais apresentam deficiências quando a característica a ser medida é do tipo acumulativa (Exemplo: Torque). Para lidar com esse tipo de característica sugere-se o uso de um método alternativo, baseado em Quadrados Latinos. São discutidos os resultados de aplicações práticas que demonstram a validade do método proposto.

\section{Palavras-chave: avaliação, sistema, medição, repetibilidade, reprodutibilidade, quadrado latino, torque.}

\section{Introdução:}

Com a intensificação da utilização do Controle Estatístico do Processo, uma atenção maior vem sendo dada à avaliação das propriedades dos sistemas de medição. Entre as diversas propriedades destacam-se

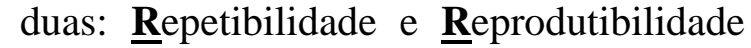
(R\&R). A avaliação de R\&R é feita mediante de estudos (AUTOMOTIVE, 1990) que detectam a influência dos operadores (Reprodutibilidade) e a influência do equi- 
pamento (Repetibilidade) sobre a variação de uma série de medições, conforme as

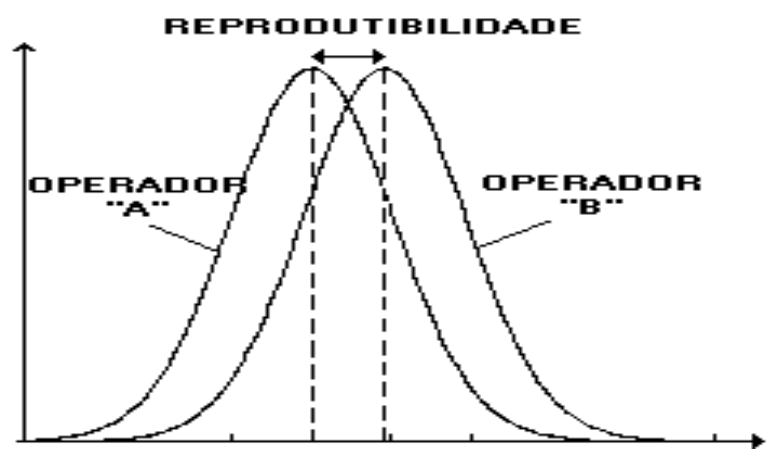

Reprodutibilidade (diferentes operadores medindo as mesmas peças).

Esses estudos vêm sendo largamente uti-lizados, mas, em determinadas situações, existe a necessidade de se adotar um mode-lo de estudo diferente, devido à presença de um acúmulo após cada medida efetuada. Por exemplo, para a medição do torque aplicado a uma junta aparafusada podemos utilizar um torquímetro; porém, devido à natureza da característica medida, figuras abaixo.

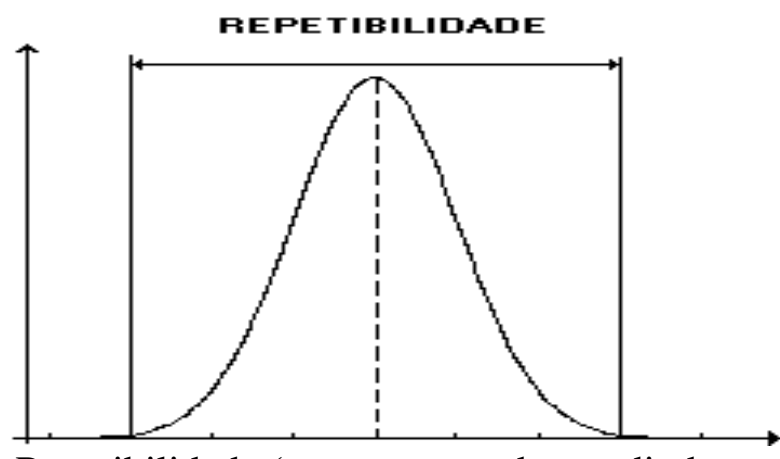

Repetibilidade (mesmo operador medindo a mesma peça diversas vezes).

a cada medição acaba-se adicionando mais torque à junta. Para uma única medição, o efeito pode ser desprezado mas para um estudo de R\&R, deve-se realizar uma série de medições sobre os mesmos pontos. Este efeito do reaperto ou acúmulo na junta acaba influenciando grandemente o resultado do estudo, como veremos a seguir.

\section{Métodos tradicionais para cálculo de $\mathbf{R} \& \mathbf{R}$ :}

Para um dado sistema de medição utilizado para avaliar uma característica, efetua-se uma série de medições organizadas de forma a permitir separar os seguintes efeitos:

- efeito das peças (VP);

- efeito dos avaliadores (VA);

- efeito do equipamento (VE);

- efeito da interação peça * avaliador (INT);
- efeito avaliador + equipamento + interação (R\&R);

- efeito total (VT).

Todos estes efeitos são medidos em termos de variância. Para efeito comparativo e definição de um critério de aceitação utiliza-se uma faixa de $\pm 2,575$ (total de 5,15) desvios-padrão, correspondendo a 99\% da área sob a curva Normal.

$$
\begin{array}{|lll|}
\hline \mathrm{VP}=5,15 * & \text { p } & \mathrm{R}^{*} \mathrm{R}^{2}=\mathrm{VA}^{2}+\mathrm{VE}^{2}+\mathrm{INT}^{2} \\
\mathrm{VA}=5,15 * & \text { a } & \\
\mathrm{VE}=5,15 * & \text { e } & \mathrm{VT}^{2}=\mathrm{R}^{2} \mathrm{R}^{2}+\mathrm{VP}^{2} \\
\mathrm{INT}=5,15 * & \text { ap } & \\
\hline
\end{array}
$$

onde:

$\mathrm{p}$ = desvio-padrão devido às peças;

$\mathrm{a}=$ desvio-padrão devido aos

operadores;

e = desvio-padrão devido ao equipamento; ap = desvio-padrão devido à interação.

Para obter as estimativas p, a, e e ap utiliza-se tradicionalmente $\mathrm{o}$ seguinte modelo: 
Para o caso de 2 operadores com 2 replicações, o estudo é realizado da seguinte forma:

Esquema das medições com os operadores "A" e "B".

\begin{tabular}{|l|c|c|c|c|c|c|c|c|c|c|}
\hline Série & 1 & 2 & 3 & 4 & 5 & 6 & 7 & 8 & 9 & 10 \\
\hline $1^{\mathrm{a}}$ medição & $\mathrm{A}$ & $\mathrm{A}$ & $\mathrm{A}$ & $\mathrm{A}$ & $\mathrm{A}$ & $\mathrm{A}$ & $\mathrm{A}$ & $\mathrm{A}$ & $\mathrm{A}$ & $\mathrm{A}$ \\
\hline $2^{\mathrm{a}}$ medição & $\mathrm{B}$ & $\mathrm{B}$ & $\mathrm{B}$ & $\mathrm{B}$ & $\mathrm{B}$ & $\mathrm{B}$ & $\mathrm{B}$ & $\mathrm{B}$ & $\mathrm{B}$ & $\mathrm{B}$ \\
\hline $3^{\mathrm{a}}$ medição & $\mathrm{A}$ & $\mathrm{A}$ & $\mathrm{A}$ & $\mathrm{A}$ & $\mathrm{A}$ & $\mathrm{A}$ & $\mathrm{A}$ & $\mathrm{A}$ & $\mathrm{A}$ & $\mathrm{A}$ \\
\hline $4^{\mathrm{a}}$ medição & $\mathrm{B}$ & $\mathrm{B}$ & $\mathrm{B}$ & $\mathrm{B}$ & $\mathrm{B}$ & $\mathrm{B}$ & $\mathrm{B}$ & $\mathrm{B}$ & $\mathrm{B}$ & $\mathrm{B}$ \\
\hline
\end{tabular}

Tendo obtido estes dados, pode-se utilizar duas formas de obter o valor de R\&R:

- Método da ANOVA (Análise da Variância);
- Método das amplitudes.

A referência AUTOMOTIVE (1990) detalha extensamente cada método utilizado e os critérios de aprovação.

\section{Método da ANOVA:}

Este é o método mais completo, porém é de cálculo mais complexo e requer maior conhecimento para a análise dos resultados. A grande vantagem está na obtenção de uma estimativa para ap (desvio-padrão das medições devido à interação peça * avalia-dor), que não é possível pelo método das amplitudes. Utilizando a técnica da Análise de Variância, monta-se um quadro do qual se obtêm as estimativas p, a, e e ap, e portanto o valor de R\&R.

\section{* Método das amplitudes:}

Este método é muito mais utilizado devido à sua simplicidade de cálculo, porém não permite obter estimativa da interação peça * avaliador. O método consiste em calcular as amplitudes entre as médias de cada operador, entre as médias de cada peça e a amplitude média de cada operador sobre cada peça. Com estas três amplitudes pode-se obter VA, VE e VP, e portanto o valor de R\&R.

\section{*ritério de aprovação:}

Pelos 2 métodos iremos obter estimativas do $R \& R$, porém tais estimativas estarão na mesma grandeza dos valores em que foram medidas (Exemplo: Lbs-pé, $\mathrm{mm}, \mathrm{Ph})$. Para estabelecer um critério, devemos divi-dir o R\&R por uma base de mesma gran-deza, obtendo um número adimensional. As tabelas a seguir trazem as bases utilizadas em cada situação e o critério de aprovação utilizado.

Bases utilizadas para cálculo das porcentagens de R\&R.

\begin{tabular}{|c|c|}
\hline SITUAÇÃO & BASE UTILIZADA \\
\hline Tolerância bilateral & Tolerância \\
\hline Processos instáveis ou incapazes & Variação Total (VT) do estudo de R\&R \\
\hline Processos estáveis e capazes & 5,15 desvios-padrão do processo \\
\hline
\end{tabular}




\begin{tabular}{|c|c|}
\hline \% ENCONTRADA & RESULTADO \\
\hline de $0 \%$ até $10 \%$ & Sistema aprovado. \\
\hline de $10 \%$ até $30 \%$ & Sistema requer melhoria mas pode ser aprovado. \\
\hline mais do que $30 \%$ & Sistema rejeitado. \\
\hline
\end{tabular}

\section{Exemplos Numéricos:}

A seguir dois exemplos de cálculo realizados pelo método das amplitudes (no pri- meiro não existe o efeito acumulativo e o segundo é um exemplo típico desse efeito).

\section{Exemplo 1: medição da folga do cubo da roda de 10 eixos de caminhões.}

Dados coletados para o estudo de R\&R (3 operadores $* 3$ repeticões).

\begin{tabular}{|c|c|c|c|c|c|c|c|c|c|c|c|}
\hline \multicolumn{2}{|c|}{ Dados: mícrons } & \multicolumn{10}{|c|}{ PEÇAS } \\
\hline Série & Operador & 1 & 2 & 3 & 4 & 5 & 6 & 7 & 8 & $\overline{9}$ & 10 \\
\hline $1^{\mathrm{a}}$ & $A$ & 8,0 & 14,0 & 18,0 & 2,0 & 12,0 & 19,0 & 6,0 & 16,5 & 22,0 & 12,0 \\
\hline $2^{a}$ & $B$ & 9,0 & 13,0 & 18,5 & 2,0 & 13,0 & 19,0 & 6,0 & 17,0 & 22,0 & 13,0 \\
\hline $3^{a}$ & $\mathrm{C}$ & 10,0 & 14,0 & 19,5 & 1,5 & 14,0 & 20,0 & 6,0 & 18,0 & 21,5 & 13,0 \\
\hline $4^{\mathrm{a}}$ & $A$ & 8,5 & 14,5 & 19,0 & 1,5 & 13,0 & 20,0 & 5,0 & 18,0 & 22,0 & 13,0 \\
\hline $5^{a}$ & $B$ & 9,0 & 12,5 & 20,0 & 1,0 & 13,0 & 20,0 & 5,0 & 17,5 & 22,0 & 12,0 \\
\hline $6^{a}$ & $\bar{C}$ & 11,0 & 15,0 & 21,0 & 1,0 & 14,0 & 20,0 & 5,0 & 17,5 & 22,0 & 13,0 \\
\hline $7^{a}$ & $\bar{A}$ & 9,5 & 14,0 & 20,0 & 1,5 & 13,0 & 20,0 & 6,0 & 17,5 & 22,0 & 13,0 \\
\hline $8^{a}$ & $\mathrm{~B}$ & 9,5 & 13,0 & 20,0 & 1,5 & 13,0 & 20,0 & 5,0 & 17,5 & 22,0 & 13,0 \\
\hline $9^{a}$ & $\mathrm{C}$ & 10,0 & 14,0 & 21,0 & 2,0 & 13,0 & 20,0 & 5,0 & 17,5 & 22,0 & 13,0 \\
\hline
\end{tabular}

Resultados do estudo de R\&R do exemplo 1.

\begin{tabular}{|lr|c|c|c|}
\hline \multicolumn{2}{|c|}{ Item } & Valor & \% de VT & \% da Tolerância \\
\hline Repetibilidade & VE & 2,49 & $7,5 \%$ & $11,1 \%$ \\
\hline Reprodutibilidade & VA & 1,27 & $3,8 \%$ & $5,6 \%$ \\
\hline & R\&R & 2,80 & $8,5 \%$ & $12,4 \%$ \\
\hline Var. das Peças & VP & 32,94 & \multicolumn{3}{l}{} \\
\hline Var. Total & VT & 33,05 & \multicolumn{1}{|l}{} \\
\hline
\end{tabular}

\section{Exemplo 2: medição do torque de fixação do conjunto de embreagem de 10 pickups.}

Dados coletados para o estudo de R\&R (2 operadores $* 2$ repetições).

\begin{tabular}{|c|c|c|c|c|c|c|c|c|c|c|c|}
\hline \multicolumn{2}{|c|}{ Newton-metro } & \multicolumn{10}{|c|}{ PEÇAS } \\
\hline Série & Operador & 1 & $\overline{2}$ & $\overline{3}$ & 4 & 5 & 6 & $\overline{7}$ & 8 & 9 & 10 \\
\hline $1^{\mathrm{a}}$ & $\bar{A}$ & $\overline{22,0}$ & 21,5 & 22,5 & 20,0 & 23,0 & $\overline{21,0}$ & $\overline{24,0}$ & $\overline{23,0}$ & 22,5 & $\overline{20,0}$ \\
\hline $2^{a}$ & $\bar{B}$ & 23,0 & 23,0 & 24,0 & 21,5 & 24,0 & 22,5 & 25,0 & 23,5 & 23,5 & 21,5 \\
\hline $3^{a}$ & $\bar{A}$ & 24,5 & 25,0 & 25,0 & 22,5 & 24,5 & 24,0 & 26,5 & 24,5 & 25,0 & 23,0 \\
\hline $4^{\mathrm{a}}$ & $B$ & 26,0 & 26,0 & 26,5 & 24,5 & 26,0 & 25,0 & 28,0 & 25,0 & 26,5 & 25,5 \\
\hline
\end{tabular}

Resultados do estudo de R\&R do exemplo 2.

\begin{tabular}{|lr|c|c|c|}
\hline \multicolumn{1}{|c|}{ Item } & Valor & \% de VT & \% da Tolerância \\
\hline Repetibilidade & VE & 11,97 & $85,4 \%$ & $171,0 \%$ \\
\hline Reprodutibilidade & VA & 4,03 & $28,7 \%$ & $57,5 \%$ \\
\hline & R\&R & 12,62 & $90,1 \%$ & $180,4 \%$ \\
\hline Var. das Peças & VP & 6,08 & \multicolumn{3}{l}{} \\
\cline { 1 - 3 } Var. Total & VT & 14,01 &
\end{tabular}


Como se vê, o efeito do "reaperto" no caso do torque inflaciona tremendamente os resultados, resultando na rejeição do sis- tema. Esse "reaperto" fica evidente se calcularmos as médias de cada linha (série de medições):

Dados e médias de cada série de medições, mostrando o efeito do "reaperto".

\begin{tabular}{|c|c|c|c|c|c|c|c|c|c|c|c|}
\hline Série Peça & 1 & 2 & 3 & 4 & 5 & 6 & 7 & 8 & 9 & 10 & Média \\
\hline $1^{\mathrm{a}}$ medição & 22,0 & 21,5 & 22,5 & 20,0 & 23,0 & 21,0 & 24,0 & 23,0 & 22,5 & 20,0 & 21,55 \\
\hline $2^{\mathrm{a}}$ medição & 23,0 & 23,0 & 24,0 & 21,5 & 24,0 & 22,5 & 25,0 & 23,5 & 23,5 & 21,5 & 23,15 \\
\hline $3^{\mathrm{a}}$ medição & 24,5 & 25,0 & 25,0 & 22,5 & 24,5 & 24,0 & 26,5 & 24,5 & 25,0 & 23,0 & 24,45 \\
\hline $4^{\mathrm{a}}$ medição & 26,0 & 26,0 & 26,5 & 24,5 & 26,0 & 25,0 & 28,0 & 25,0 & 26,5 & 25,5 & 25,90 \\
\hline
\end{tabular}

O método tradicional revela-se inadequado para lidar com essa situação, sendo portanto necessário buscar-se um novo modelo.

\section{Método dos Quadrados Latinos:}

Evidencia-se no caso anterior a presença de uma variável (ordem) além das já citadas (peças, operadores e equipamento). Há necessidade, portanto, da adoção de um modelo que permita separar estes efeitos.

A influência do equipamento estará presente em todas as medições e portanto será avaliada pela variação residual, ou seja, a par-te da variância não explicada pelas demais va-riáveis (peças, operadores, ordem). Um mo-delo que poderia ser usado para a separação dos efeitos e de suas interações seria um delineamento fatorial completo, porém, dada a natureza da variável “ordem” não é possível realizar todas as combinações necessárias (Exemplo: ao medir a peça "1" com o opera-dor "A" pela "primeira" vez, torna-se impos-sível medir novamente a peça “ 1 ” com o operador "B” pela "primeira” vez. Neste caso já perdemos a "primeira” vez e esta é a "se- gunda”). Para resolver este problema precisamos adotar a "blocagem" das variáveis. Um modelo que permite simultaneamente bloquear 2 fontes de variação, além do tratamento principal, é o delineamento do tipo Quadrado Latino. Este modelo tem a desvantagem de pressupor a não existência de interação entre as variáveis, o que não parece ser um grande empecilho devido à natureza das variáveis (peça * ordem, peça * operador, operador * ordem). Isso poderá ser compro-vado na análise dos resultados.

Quadrados Latinos existem para tamanhos “p 2” e consistem na colocação de uma va-riável com diferentes níveis a cada linha, uma com diferentes níveis a cada coluna e uma com diferentes níveis a cada letra do alfabeto latino, de forma a manter o mesmo número de níveis para as três variáveis.

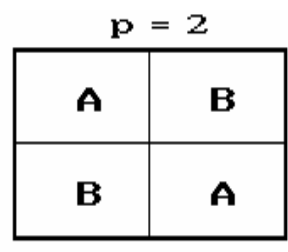

\begin{tabular}{|c|c|c|}
\multicolumn{1}{c}{$\mathbf{p = 3}$} \\
\hline A & B & C \\
\hline B & C & A \\
\hline C & A & B \\
\hline
\end{tabular}

\begin{tabular}{|c|c|c|c|}
\hline \multicolumn{5}{|c|}{$\mathbf{P}=\mathbf{4}$} \\
\hline A & B & C & D \\
\hline C & D & D & A \\
\hline D & A & B & B \\
\hline
\end{tabular}

Exemplos de Quadrados Latinos ( $\mathrm{p}=2 ; 3 ; 4)$

Pode-se notar que cada tratamento (letra) aparece uma única vez em cada coluna e uma única vez em cada linha. No nosso caso decidimos utilizar uma estrutura 
com “p = 3” pois tradicionalmente este é o número de operadores utilizados num estudo de sistemas de medição. Neste caso as linhas representam a ordem, as colunas representam as peças e as letras os operadores:

\begin{tabular}{|c|c|c|c|}
\hline ardem pera & 1 & 2 & 3 \\
\hline 1 트 & $\mathbf{A}$ & $\mathbf{B}$ & C \\
\hline $2^{\mathrm{B}}$ & B & $C$ & $\mathbf{A}$ \\
\hline 3르 & $C$ & $\mathbf{A}$ & B \\
\hline
\end{tabular}

Estrutura básica do modelo adotado (cada letra representa um operador).

Intuitivamente podemos perceber que se calcularmos as médias de cada linha (primeira, segunda e terceira), estas médias terão todas o efeito somado dos operadores A, B e C e das peças 1,2 e 3, ou seja, se houver diferença significativa entre estas médias das linhas, esta não poderá ser atribuída às variáveis operador e peças pois ambas agem igualmente na primeira, segun-da e terceira linhas (o que não ocorria no modelo tradicional de $\mathrm{R} \& \mathrm{R})$. $\mathrm{O}$ mesmo raciocínio pode ser estendido para os ope-radores e as peças, avaliando-se as médias das letras e das colunas. Temos agora, por-tanto, como separar os efeitos das variáveis operador, peças e ordem, sendo que o do equipamento será mensurado pelo resíduo.

Estatisticamente temos o seguinte modelo:

$$
\mathrm{Y}_{\mathrm{ijk}}=+\mathrm{i}+\mathrm{j}+\mathrm{k}+\mathrm{ijk} \quad \begin{aligned}
& \mathrm{i}=1,2, \ldots \mathrm{p} \\
& \mathrm{j}=1,2, \ldots \mathrm{p} \\
& \mathrm{k}=1,2, \ldots \mathrm{p}
\end{aligned}
$$

onde:

$\mathrm{Y}_{\mathrm{ijk}}=$ observação na linha $\mathrm{i}$, coluna $\mathrm{j}$,

letra $\mathrm{k}$;

$\mathrm{m}=$ média global;

$\mathrm{a}_{\mathrm{i}}=$ efeito da linha “i” (ordem);

$b_{j}=$ efeito da coluna “j” (peças); $\mathrm{g}_{\mathrm{k}}=$ efeito da letra " $\mathrm{k}$ " (avaliador);

$\mathrm{E}_{\mathrm{ijk}}=$ erro “ijk” (resíduo/equipamento);

A análise de variância consistirá em par-ticionar a soma total de quadrados das $\mathrm{N}=\mathrm{p}$ observações nos componentes letras, colunas, linhas e resíduo.

$$
\mathrm{SQ}_{\text {total }}=\mathrm{SQ}_{\text {linhas }}+\mathrm{SQ}_{\text {colunas }}+\mathrm{SQ}_{\text {letras }}+\mathrm{SQ}_{\text {erro }}
$$

ou

$$
\mathrm{SQT}=\mathrm{SQO}+\mathrm{SQP}+\mathrm{SQA}+\mathrm{SQE}
$$

Uma desvantagem de se utilizar um Quadrado Latino pequeno ( $p=3$ ou 4) é o pequeno número de graus de liberdade do resíduo (para $\mathrm{p}=3$ temos apenas 2 g.l. para o resíduo). Isso pode ser resolvido ado-tando-se diversos Quadrados Latinos (re-plicações). No nosso caso optamos por re-plicar utilizando os mesmos operadores e ordem, tomando novas peças, totalizando 5 Quadrados Latinos:

Esquema das medições com os operadores A, B e C. 


\begin{tabular}{|c|c|c|c|c|c|c|c|c|c|c|c|c|c|c|c|}
\hline & 1 & 2 & 3 & 4 & 5 & 6 & 7 & 8 & 9 & 10 & 11 & 12 & 13 & 14 & 15 \\
\hline $1^{\mathrm{a} m}$ & $\bar{A}$ & $\mathrm{~B}$ & $\mathrm{C}$ & $B$ & $\bar{C}$ & A & C & $\bar{A}$ & $\bar{D}$ & 4 & $B$ & $\mathrm{C}$ & $B$ & $C$ & $\bar{A}$ \\
\hline $2^{\mathrm{a}}$ medição & $\mathrm{B}$ & $\mathrm{C}$ & $A$ & C & A & $D$ & $A$ & 5 & $C$ & $\mathrm{C}$ & $A$ & $\bar{B}$ & $A$ & B & $\mathrm{C}$ \\
\hline $3^{a}$ medição & $\bar{C}$ & $\mathrm{~A}$ & $B$ & $A$ & $\mathrm{~B}$ & - & $\mathrm{B}$ & $C$ & A & $\mathrm{B}$ & $\mathrm{C}$ & A & $\bar{C}$ & $\mathrm{~A}$ & \\
\hline
\end{tabular}

Para adicionar mais aleatoriedade ao estudo deve-se utilizar diferentes Quadrados Latinos do mesmo tamanho (para $\mathrm{p}=3$ existem 12 Quadrados diferentes). Neste caso, o quadro de Análise de Variância será:

\begin{tabular}{|c|c|c|c|c|}
\hline $\begin{array}{l}\text { Fonte de } \\
\text { Va riaçã o }\end{array}$ & $\begin{array}{c}\text { Somade } \\
\text { Quadrados }\end{array}$ & $\begin{array}{l}\text { Graus de } \\
\text { Liberdade }\end{array}$ & $\begin{array}{c}\text { Quadrado } \\
\text { M éd io }\end{array}$ & $\begin{array}{c}\mathrm{F} \\
\mathrm{c} \mathrm{a} \mathrm{lc} \mathrm{u} \mathrm{la} \mathrm{do}\end{array}$ \\
\hline Orde m & S Q O & 2 & $\mathrm{QO}=\frac{\mathrm{SQO}}{2}$ & Qo $Q$ \\
\hline $\mathrm{P}$ e ç a & S Q P & 14 & $Q p=\frac{S Q P}{14}$ & $\mathrm{Qp}$ \\
\hline A $v$ a lia $d$ or & S Q A & 2 & $Q \mathrm{a}=\frac{\mathrm{SQA}}{2}$ & $\mathrm{Q} a$ \\
\hline Equip a mento & S Q E & 26 & $\mathrm{Qe}=\frac{\mathrm{SQE}}{26}$ & \\
\hline Total & S Q T & 44 & & \\
\hline
\end{tabular}

Quadro de Análise de Variância (ANOVA).

onde:

\begin{tabular}{|c|c|}
\hline SQO $=\sum_{i=1}^{3} \frac{T_{i}^{2}}{15}-\frac{T^{2}}{45}$ & $\operatorname{SQP}=\sum_{J=1}^{15} \frac{T_{J}^{2}}{3}-\frac{T^{2}}{45}$ \\
\hline SQA $=\sum_{K=A}^{C} \frac{T_{K}^{2}}{15}-\frac{T^{2}}{45}$ & SQT $=Q-\frac{T^{2}}{45}$ \\
\hline \multicolumn{2}{|c|}{ SQE $=$ SQT $-(S Q O+S Q P+S Q A)$} \\
\hline
\end{tabular}

Fórmulas utilizadas na Análise de Variância.

e:

$$
\begin{aligned}
& \mathbf{T}_{\mathrm{i}}=\text { soma dos valores da linha “i”" }(\mathrm{i}=1,2,3) \\
& \mathbf{T}_{\mathrm{j}}=\text { soma dos valores da coluna “ } \mathrm{j} "(\mathrm{j}=1,2 \ldots, 15) \\
& \mathbf{T}_{\mathrm{k}}=\text { soma dos valores da letra } \mathrm{k} \text { " }(\mathrm{k}=\mathrm{A}, \mathrm{B}, \mathrm{C}) \\
& \mathbf{T}=\text { soma de todos os valores } \\
& \mathbf{Q}=\text { soma dos quadrados de todos os valores }
\end{aligned}
$$

Pelo modelo adotado, o quadrado médio esperado para cada variável será: 


\begin{tabular}{|lll|}
\hline ORDEM & $:$ & $\mathrm{Q}_{\mathrm{o}}=\mathrm{e}^{2}+3 * \mathrm{o}^{2}$ \\
AVALIADOR & $:$ & $\mathrm{Q}_{\mathrm{a}}=\mathrm{e}^{2}+3 *{ }^{2}$ \\
PEÇA & $:$ & $\mathrm{Q}_{\mathrm{p}}=\mathrm{e}^{2}+15 *{ }^{2}$ \\
EQUIPAMENTO & $:$ & $\mathrm{Q}_{\mathrm{e}}=\mathrm{e}^{2}$ \\
\hline
\end{tabular}

Portanto, quando dividimos estes quadrados médios pelo erro ou resíduo (equipamento) devemos obter:

\begin{tabular}{|l|}
\hline$\frac{Q_{\mathrm{o}}}{\mathrm{Q}_{\mathrm{e}}}=1+\frac{3^{*} \sigma_{\mathrm{o}}^{2}}{\sigma_{\mathrm{e}}^{2}}$ \\
\hline$\frac{\mathrm{Q}_{\mathrm{a}}}{\mathrm{Q}_{\mathrm{e}}}=1+\frac{3^{*} \sigma_{\mathrm{a}}^{2}}{\sigma_{\mathrm{e}}^{2}}$ \\
\hline$\frac{\mathrm{Q}_{\mathrm{p}}}{\mathrm{Q}_{\mathrm{e}}}=1+\frac{15^{*} \sigma_{\mathrm{p}}^{2}}{\sigma_{\mathrm{e}}^{2}}$ \\
\hline
\end{tabular}

Intuitivamente vemos que os valores $\mathrm{F}_{\text {calc }}$ serão tão maiores que 1 quanto mais significativa for a variável em questão, ou seja, quanto maior for o quociente entre a variância em análise e a variância residual. $E$ É evidente que quando $\mathrm{F}_{\text {calc }}<1$, isto só pode ser atribuído ao acaso. Numa Análise de Variância, tradicionalmente testa-se a significância das variáveis envolvidas a um determinado nível pré-estabelecido (geralmente $5 \%$ ou $1 \%$ ). Quando se rejeita a hipótese testada (ou seja, quando há evidência da significância da correspondente variável) obviamente o modelo não se altera, podendo-se obter as estimativas de todos os desvios-padrão necessários ao cálculo de R\&R. Porém, nada se pode afirmar quando a hipótese é aceita, ou seja, quando não há evidência de que determinada variável seja significativa (o que não equivale a afirmar que há evidência de que determi-nada variável não é significativa).
Se tivermos razões para supor uma variável como não significativa podemos acres-centar sua soma de quadrados e graus de liberdade ao resíduo e refazer a análise de variância. A referência AUTOMOTIVE (1990) sugere utilizar um nível de 25\% de significância mas PAULL (1950) demonstra ser melhor utilizar o critério de $\mathrm{F}_{\text {calc }}<2 * \mathrm{~F}_{50 \%,{ }_{1}, 2}$ e portanto adotaremos este procedimento neste estudo (“ 1 ” $\mathrm{e}$ “ 2 ” são os graus de liberdade do numerador e do denominador, respectivamente), substituindo o critério tradicional da Análise de Variância com base nos níveis de significância usuais pela idéia de se considerar significativo o efeito da variável, de acordo com o procedimento acima mencionado.

Tendo concluído quanto à significância ou não das variáveis, podemos então calcular as estimativas de o, a, p e e, necessárias para o cálculo de R\&R. Temos:

$$
\begin{array}{|c|}
\hline \mathrm{e}^{2}=\mathrm{Q}_{\mathrm{e}} \\
\mathrm{o}^{2}=\left(\mathrm{Q}_{\mathrm{o}}-\mathrm{Q}_{\mathrm{e}}\right) / 3 \\
\mathrm{a}^{2}=\left(\mathrm{Q}_{\mathrm{a}}-\mathrm{Q}_{\mathrm{e}}\right) / 3 \\
\mathrm{p}^{2}=\left(\mathrm{Q}_{\mathrm{p}}-\mathrm{Q}_{\mathrm{e}}\right) / 15
\end{array}
$$

Novamente temos: 


\begin{tabular}{|l|}
$\mathrm{VP}=5.15 *$ \\
$\mathrm{VA}=5.15 *$ \\
$\mathrm{VE}=5.15 *$ \\
\hline
\end{tabular}

e mais:

$$
\mathrm{VO}=5.15 * \text { o }
$$

Não há estimativa da interação pois esta foi considerada inexistente.

\begin{tabular}{c}
$\mathrm{R} \& \mathrm{R}^{2}=\mathrm{VA}^{2}+\mathrm{VE}^{2}$ \\
$\mathrm{VT}^{2}=\mathrm{R}^{2} \mathrm{R}^{2}+\mathrm{VP}^{2}+\mathrm{VO}^{2}$ \\
\hline
\end{tabular}

Como se vê, o método dos Quadrados Latinos também se utiliza da técnica de Análise de Variância para obter os resulta- dos, mas distingue-se do método tradicionalmente chamado de "ANOVA" pelo arranjo das medições.

\section{Exemplo numérico:}

Medição: Torque (Nm) da porca da caixa de câmbio (F-1000).

\begin{tabular}{|c|c|c|c|c|c|c|c|c|c|c|c|c|c|c|c|}
\hline & 1 & 2 & 3 & 4 & 5 & 6 & 7 & 8 & 9 & 10 & 11 & 12 & 13 & 14 & 15 \\
\hline $\begin{array}{l}\text { Operador } \\
1^{\mathrm{a}} \text { medição }\end{array}$ & $\begin{array}{c}\mathrm{A} \\
42,5\end{array}$ & $\begin{array}{c}\text { B } \\
145,0\end{array}$ & $\begin{array}{c}C \\
142,5\end{array}$ & $\begin{array}{c}\mathrm{B} \\
132,5\end{array}$ & $\begin{array}{c}\mathrm{C} \\
145,0\end{array}$ & $\begin{array}{c}A \\
140,0\end{array}$ & $\begin{array}{c}C \\
135,0\end{array}$ & $\begin{array}{c}A \\
135,0\end{array}$ & $\begin{array}{c}\mathrm{B} \\
135,0\end{array}$ & $\begin{array}{c}\mathrm{A} \\
140,0\end{array}$ & $\begin{array}{c}\text { B } \\
130,0\end{array}$ & $\begin{array}{c}C \\
135,0\end{array}$ & $\begin{array}{c}\mathrm{B} \\
132,5\end{array}$ & $\begin{array}{c}\mathrm{C} \\
135,0\end{array}$ & $\begin{array}{c}A \\
132,5\end{array}$ \\
\hline $\begin{array}{l}\text { Operador } \\
3^{\mathrm{a}} \text { medição }\end{array}$ & $\begin{array}{c}C \\
150,0\end{array}$ & $\begin{array}{c}\mathrm{A} \\
147,5\end{array}$ & \begin{tabular}{|c|}
$B$ \\
145,0
\end{tabular} & \begin{tabular}{|c|}
$A$ \\
140,0
\end{tabular} & \begin{tabular}{|c|}
$\mathrm{B}$ \\
152,5
\end{tabular} & $\begin{array}{c}C \\
150,0\end{array}$ & \begin{tabular}{|c|}
$B$ \\
140,0
\end{tabular} & $\begin{array}{c}C \\
142,5\end{array}$ & $\begin{array}{c}A \\
140,0\end{array}$ & $\begin{array}{c}\mathrm{B} \\
147,5\end{array}$ & $\begin{array}{c}C \\
135,0\end{array}$ & $\begin{array}{c}\mathrm{A} \\
142,5\end{array}$ & $\begin{array}{c}C \\
140,0\end{array}$ & \begin{tabular}{|c|}
$A$ \\
140,0
\end{tabular} & $\begin{array}{c}B \\
140,0\end{array}$ \\
\hline
\end{tabular}

Quadro inicial da Análise de Variância, onde se nota que o efeito dos avaliadores não é significativo ( $\left.\mathrm{F}_{\text {calculado }}<\mathrm{F}_{\text {limite }}\right)$

\begin{tabular}{|r|c|c|c|c|c|}
\hline $\begin{array}{c}\text { Fonte de } \\
\text { Variação }\end{array}$ & $\begin{array}{c}\text { Soma de } \\
\text { Quadrados }\end{array}$ & $\begin{array}{c}\text { Graus de } \\
\text { Liberdade }\end{array}$ & $\begin{array}{c}\text { Quadrado } \\
\text { Médio }\end{array}$ & $\begin{array}{c}\mathrm{F} \\
\text { calculado }\end{array}$ & $\begin{array}{c}\mathrm{F} \\
\text { limite }\end{array}$ \\
\hline Ordem & 300,83 & 2 & 150,42 & 93,86 & 1,42 \\
Peça & 1011,67 & 14 & 72,26 & 45,09 & 1,96 \\
Avaliador & 3,33 & 2 & 1,67 & 1,04 & 1,42 \\
Equipamento & 41,67 & 26 & 1,60 & & \\
\hline Total & 1357,50 & 44 & & & \\
\hline
\end{tabular}

Quadro final da Análise de Variância para o exemplo numérico.

\begin{tabular}{|r|r|c|c|c|c|c|}
\hline $\begin{array}{c}\text { Fonte de } \\
\text { Variação }\end{array}$ & $\begin{array}{c}\text { Soma de } \\
\text { Quadrados }\end{array}$ & $\begin{array}{c}\text { Graus de } \\
\text { Liberdade }\end{array}$ & $\begin{array}{c}\text { Quadrado } \\
\text { Médio }\end{array}$ & $\begin{array}{c}\mathrm{F} \\
\text { calculado }\end{array}$ & $\begin{array}{c}\mathrm{F} \\
\text { limite }\end{array}$ & $\begin{array}{c}\text { Desvio } \\
\text { Padrão }\end{array}$ \\
\hline Ordem & 300,83 & 2 & 150,42 & 93,59 & 1,42 & 7,04 \\
Peça & 1011,67 & 14 & 72,26 & 44,96 & 1,95 & 2,17 \\
Equipamento & 45,00 & 28 & 1,61 & & & 1,27 \\
\hline Total & 1357,50 & 44 & & & & \\
\hline
\end{tabular}

Resultados do estudo com Quadrados Latinos. 


\begin{tabular}{|c|c|c|c|c|c|}
\hline & \multicolumn{2}{|l|}{ A } & B & C & D \\
\hline 1 & \multicolumn{2}{|l|}{ Item } & Valor & $\%$ de VT & \% da Tolerância \\
\hline 2 & Repetibilidade & VE & 6,53 & $17,0 \%$ & $24,2 \%$ \\
\hline 3 & Reprodutibilidade & VA & 0,00 & $0,0 \%$ & $0,0 \%$ \\
\hline 4 & & R\&R & 6,53 & $17,0 \%$ & $24,2 \%$ \\
\hline 5 & Var. das Peças & VP & 11,18 & & \\
\hline 6 & Var. da Ordem & $\mathrm{VO}$ & 36,27 & & \\
\hline 7 & Var. Total & VT & 38,51 & & \\
\hline
\end{tabular}

Portanto, pelo critério adotado, o sistema pode ser aprovado mas requer melhorias.

\section{Análise dos resultados:}

No modelo adotado existem algumas hipóteses básicas:

- distribuição Normal da característica;

- todas as populações com mesma variância residual (homocedasticidade);

- resíduos com distribuição Normal (0, $\left.\mathrm{e}^{2}\right)$;

- não existência de interação entre as variáveis.
A técnica de Análise de Variância, porém, é suficientemente robusta para permitir algum afastamento dessas hipóteses com razoável aproximação dos resultados. MONTGOMERY (1983) sugere a técnica de calcular e analisar graficamente os resíduos do modelo, para verificar se as hipóteses são válidas. Para o modelo de Quadrados Latinos os resíduos podem ser obtidos da seguinte forma:

$$
E_{i j k}=Y_{i j k}-\bar{Y}_{i . .}-\bar{Y}_{j .}-\bar{Y}_{. k}+2 * \bar{Y}_{.}
$$

onde:

$\mathrm{E}_{\mathrm{ijk}}=$ resíduo da posição "i,j,k"

$\mathrm{Y}_{\mathrm{ijk}}=$ valor medido na posição "i,j,k"

$\overline{\mathrm{Y}}_{\mathrm{i} . .}=$ média da linha "i"

$$
\begin{aligned}
& \bar{Y}_{. j .}=\text { média da coluna "j" } \\
& \bar{Y}_{. . k}=\text { média da letra "k" } \\
& \bar{Y}_{. .}=\text {média total }
\end{aligned}
$$

\begin{tabular}{|c|c|c|c|c|c|c|c|c|c|c|c|c|c|c|c|}
\hline & 1 & 2 & 3 & 4 & 5 & 6 & 7 & 8 & 9 & 10 & 11 & 12 & 13 & 14 & 15 \\
\hline $\begin{array}{l}\text { Operador } \\
\text { Resíduo }\end{array}$ & $\begin{array}{c}A \\
-0,67\end{array}$ & $\begin{array}{c}B \\
2,33\end{array}$ & $\begin{array}{c}C \\
2,00\end{array}$ & $\begin{array}{c}B \\
-0,17\end{array}$ & $\begin{array}{c}C \\
-1,33\end{array}$ & $\begin{array}{c}\text { A } \\
-1,50\end{array}$ & $\begin{array}{c}C \\
0,33\end{array}$ & $\begin{array}{c}A \\
0,17\end{array}$ & $\begin{array}{c}B \\
0,67\end{array}$ & $\begin{array}{c}\text { A } \\
-0,67\end{array}$ & $\begin{array}{c}B \\
0,67\end{array}$ & $\begin{array}{c}C \\
-1,33\end{array}$ & $\begin{array}{c}\text { B } \\
-1,00\end{array}$ & $\begin{array}{c}C \\
1,17\end{array}$ & $\begin{array}{c}A \\
-0,67\end{array}$ \\
\hline Operador & $B$ & $C$ & $A$ & $C$ & $A$ & $B$ & $A$ & $B$ & $\mathrm{C}$ & $\mathrm{C}$ & $A$ & $B$ & $A$ & $B$ & $C$ \\
\hline Resíduo & 0,83 & $-1,17$ & $-0,50$ & $-0,17$ & 1,17 & 0,00 & 0,33 & $-0,83$ & $-0,33$ & 0,50 & 0,33 & $-0,83$ & 1,17 & $-1,67$ & 0,50 \\
\hline Operador & $C$ & $A$ & $B$ & $A$ & $B$ & $C$ & $B$ & $C$ & $A$ & $B$ & $C$ & $A$ & $C$ & $A$ & $B$ \\
\hline Resíduo & 0,17 & $-1,17$ & $-1,50$ & 1,33 & 0,17 & 1,50 & 0,67 & 0,67 & $-0,33$ & 0,17 & $-1,00$ & 0,50 & $-0,17$ & 0,50 & 0,17 \\
\hline
\end{tabular}

Para o exemplo da porca da caixa de câmbio temos os seguintes resíduos:

Resíduos obtidos no estudo da porca da caixa de câmbio.

Plotando os resíduos em relação às variáveis envolvidas temos: 


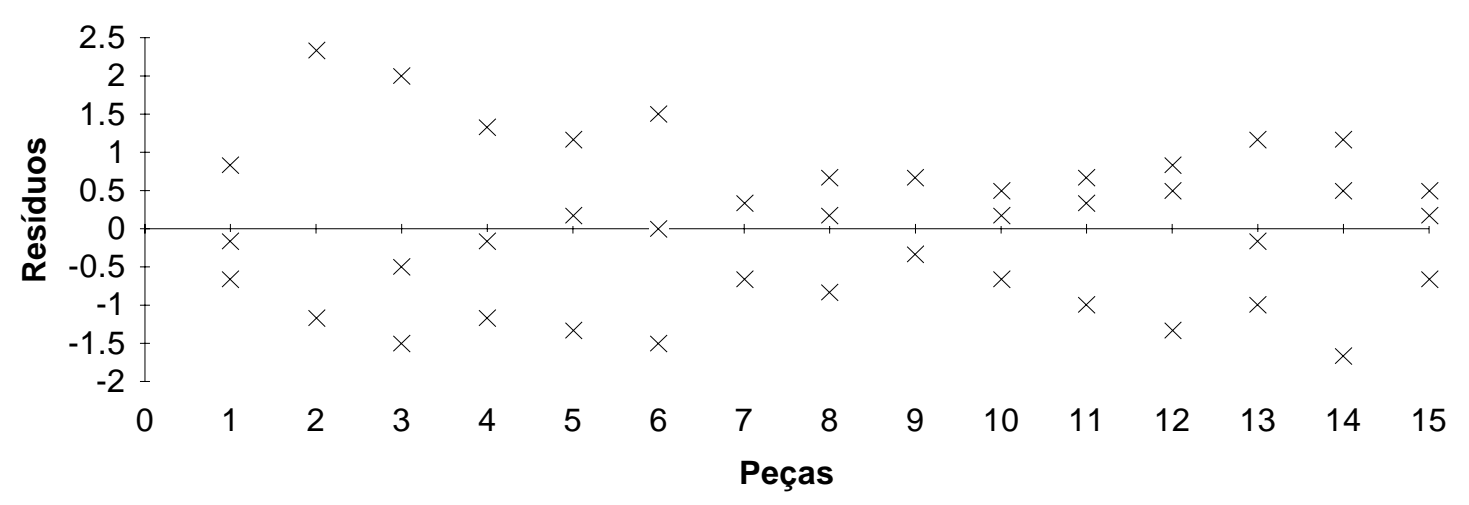

Gráfico dos Resíduos * Peças.

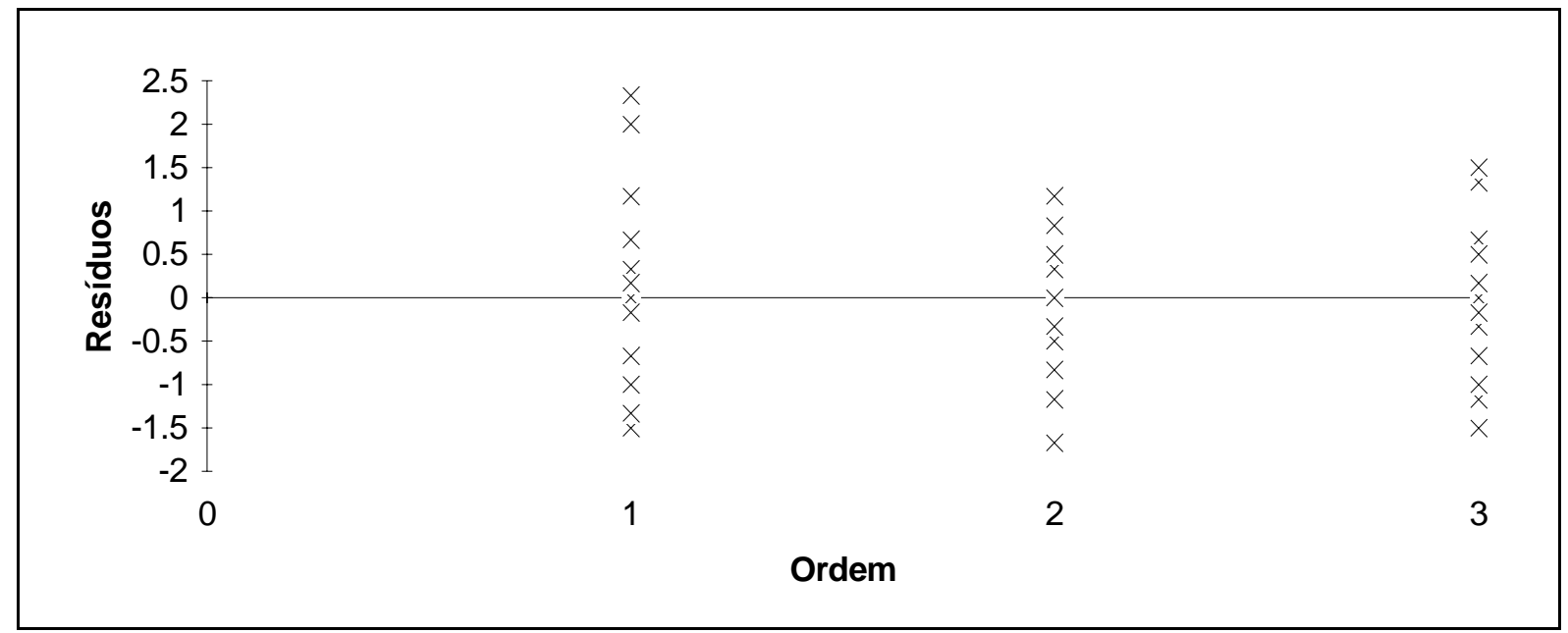

Gráfico dos Resíduos * Ordem.

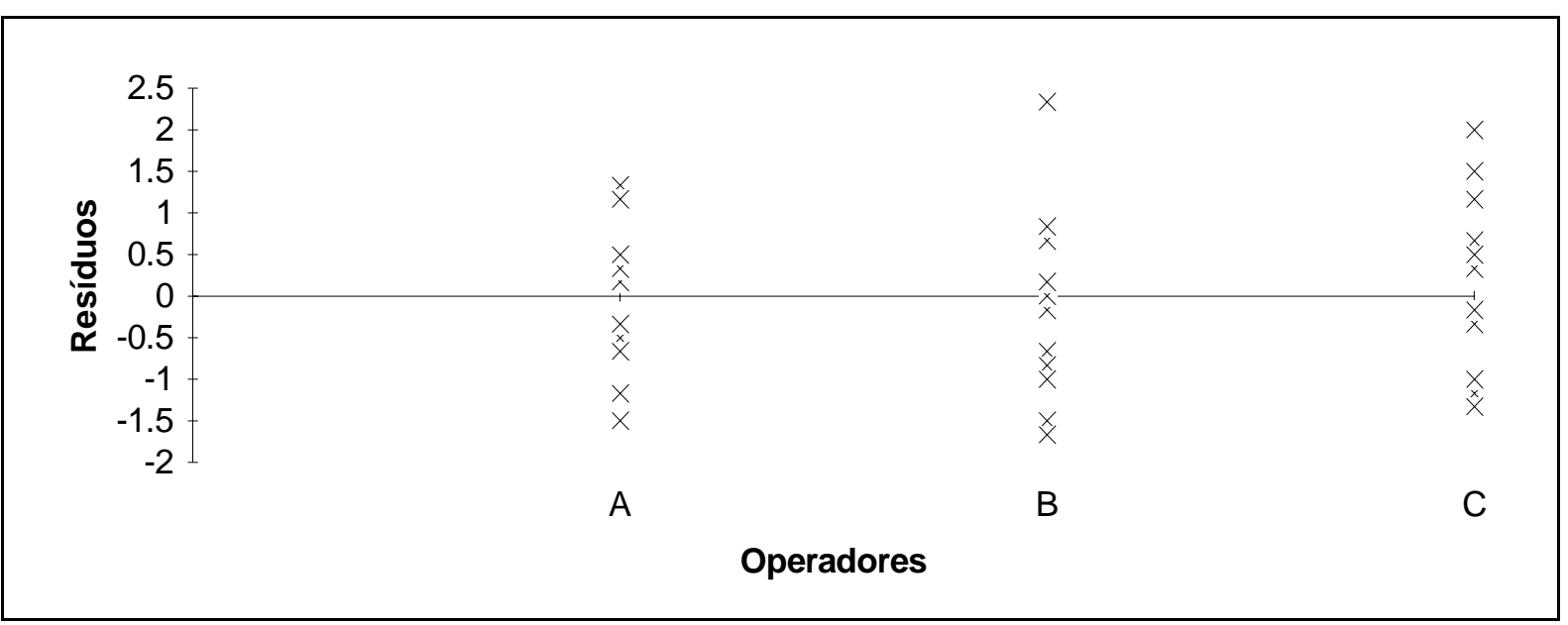

Gráfico dos Resíduos * Operadores.

Podemos também plotar os resíduos encontrados contra os valores previstos pelo modelo: 


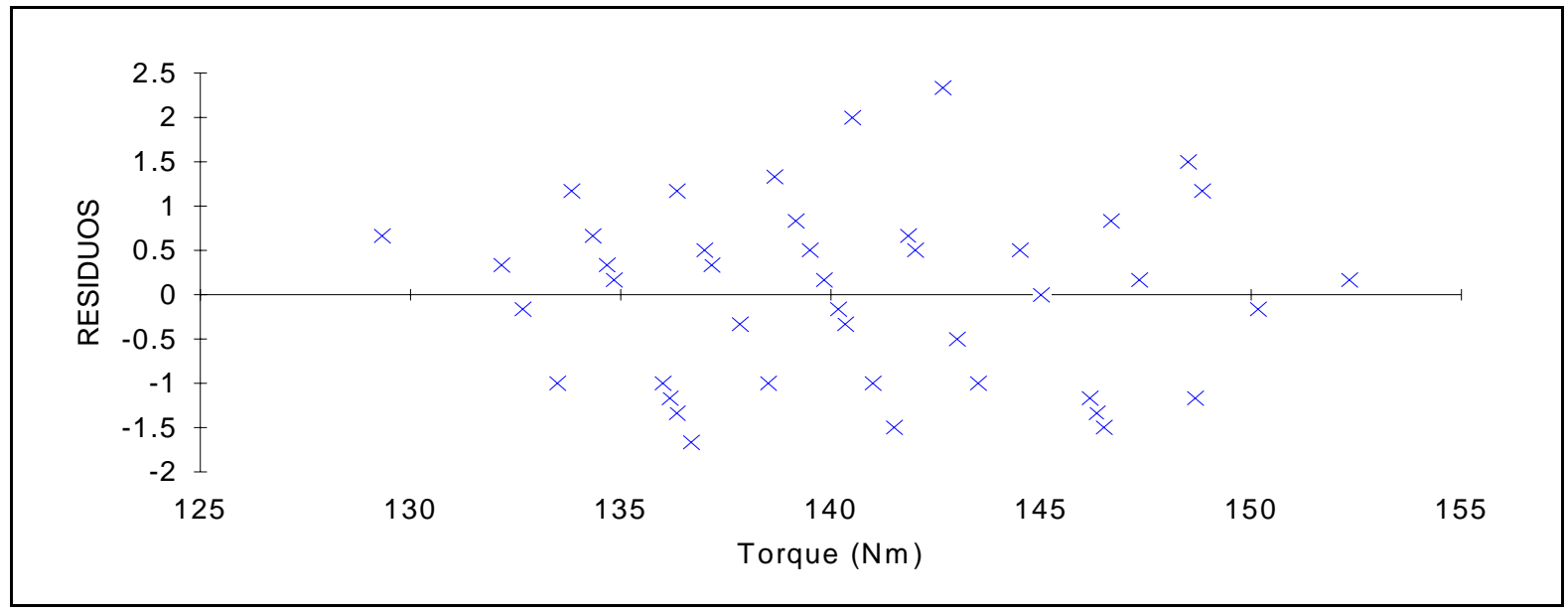

Gráfico dos Resíduos * Valores previstos pelo modelo.

Ordenando os resíduos e calculando os median-ranks (anexo 1), podemos plotar os resíduos num papel de probabilidade Normal. Uma alternativa para não necessitar do papel Normal (que possui escala nãolinear) é plotar os resíduos contra a variável "Z” (Normal padronizada) correspondente a ca-da “median-rank":

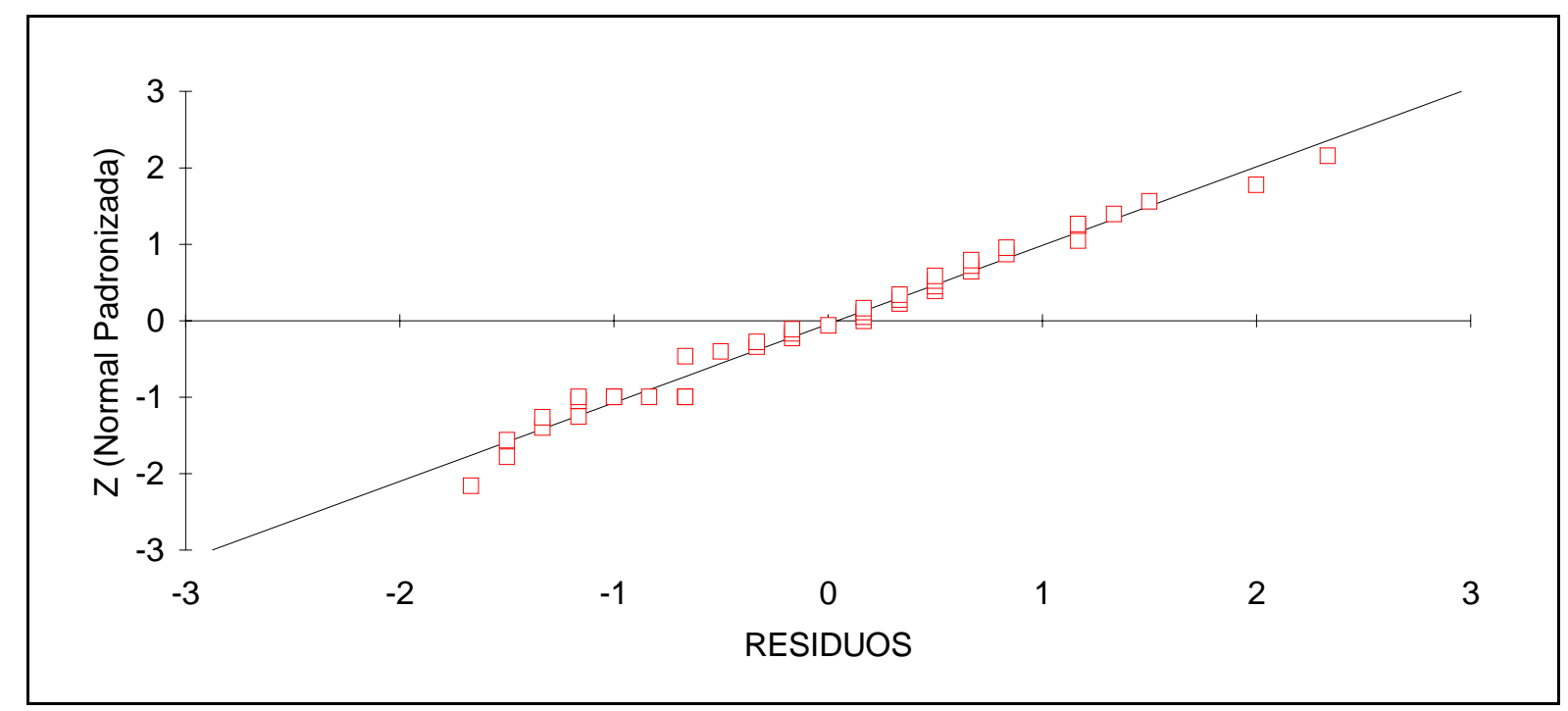

Gráfico dos Resíduos * Z (Normal Padronizada).

Pelos gráficos percebe-se que não há nenhuma tendência ou comportamento estranho dos resíduos e que há uma boa aproximação para a reta Normal. Conclui-se, então, que as hipóteses básicas são válidas e o modelo adotado é adequado.
Além de ser adequado, o modelo, ao separar o efeito da ordem do das outras variáveis, permite uma estimativa realista do valor de R\&R. Alguns resultados comparativos:

Exemplos comparativos entre o método das Amplitudes e o dos Quadrados Latinos. 


\begin{tabular}{|c|c|c|c|c|c|c|c|}
\hline $\begin{array}{l}\text { Torque de } \\
\text { Fxaçãão de: }\end{array}$ & $\begin{array}{l}\text { \%de R\&R } \\
\text { sobre: }\end{array}$ & $\begin{array}{l}\text { Método das } \\
\text { Amplitudes }\end{array}$ & $\begin{array}{l}\text { Quadrados } \\
\text { Latinos }\end{array}$ & $\begin{array}{l}\text { Torque de } \\
\text { Fxaçãa de: }\end{array}$ & $\begin{array}{l}\text { \%deR\&R } \\
\text { sobre: }\end{array}$ & $\begin{array}{l}\text { Método das } \\
\text { Amplitudes }\end{array}$ & $\begin{array}{l}\text { Quadrados } \\
\text { Latinos }\end{array}$ \\
\hline \multirow{2}{*}{$\begin{array}{l}\text { Disco ePlatô } \\
\text { de Entbreagem }\end{array}$} & Tderância & $180 \%$ & $35 \%$ & \multirow{2}{*}{$\begin{array}{l}\text { Manga } \\
\text { de eixo }\end{array}$} & Tolerância & $94 \%$ & $43 \%$ \\
\hline & $\mathrm{VT}$ & $90 \%$ & $19 \%$ & & $\overline{\mathrm{VT}}$ & $66 \%$ & $22 \%$ \\
\hline \multirow{2}{*}{$\begin{array}{l}\text { Porcada } \propto \\
\text { de Câmbio }\end{array}$} & Tderância & $93 \%$ & $24 \%$ & \multirow{2}{*}{$\begin{array}{c}\text { Válulasensível } \\
\text { à carga }\end{array}$} & Tolerância & $141 \%$ & $\overline{79 \%}$ \\
\hline & VT & $90 \%$ & $19 \%$ & & VT & $67 \%$ & $28 \%$ \\
\hline \multirow{2}{*}{$\begin{array}{c}\text { Fechadura da } \\
\text { Porta D }\end{array}$} & Tderância & $77 \%$ & $37 \%$ & \multirow{2}{*}{$\begin{array}{l}\text { Alavanca do } \\
\text { Freio Estacion. }\end{array}$} & Tolerância & $55 \%$ & $24 \%$ \\
\hline & VT & $55 \%$ & $27 \%$ & & VT & $88 \%$ & $39 \%$ \\
\hline \multirow{2}{*}{$\begin{array}{l}\text { Fechadura da } \\
\text { PortaLF }\end{array}$} & Tderância & $103 \%$ & $66 \%$ & \multirow{2}{*}{$\begin{array}{l}\text { Conjunto de } \\
\text { Ancoragem }\end{array}$} & Tolerância & $162 \%$ & $64 \%$ \\
\hline & VT & $67 \%$ & $52 \%$ & & VT & $92 \%$ & $29 \%$ \\
\hline
\end{tabular}

O gráfico a seguir mostra os resultados de 15 estudos realizados pelo método das amplitudes e 41 estudos pelo método dos
Quadrados Latinos, todos sobre operações de torque:

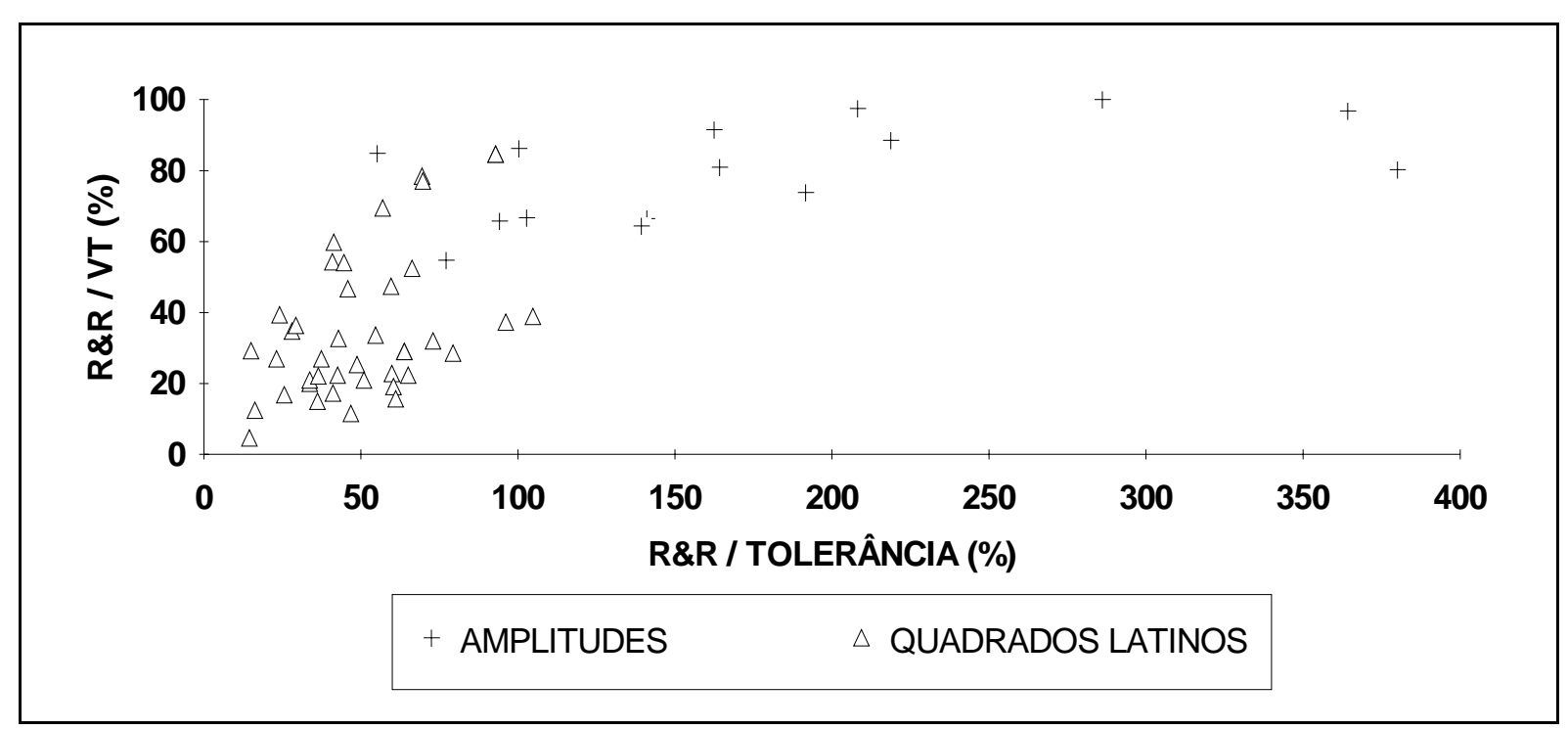

Gráfico comparativo dos dois métodos (Amplitudes e Quadrados Latinos).

Pode-se perceber que o método das amplitudes sistematicamente superestima os quocientes R\&R/Tolerância ou R\&R/VT; isso acontece porque esse método não leva em conta o efeito da ordem de medição. $\mathrm{O}$ método dos Quadrados Latinos, por outro lado, considera o efeito da ordem de medição e assim fornece estimativas confiá- veis dos quocientes R\&R/Tolerância e $\mathrm{R} \& \mathrm{R} / \mathrm{VT}$.

Uma análise residual completa é muito útil para se testar a validade do modelo, po-rém, como o utilizamos para situações mui-to semelhantes adotamos como simplifica-ção plotar apenas "resíduos * valores pre-vistos" e "resíduos * \% papel Normal”.

\section{Conclusão:}

Este trabalho discute a variabilidade de Sistemas de Medição. Tradicionalmente, pe-ças, operadores e equipamento são as fon-tes de variabilidade consideradas. Contudo, em determinadas situações percebe-se um acúmulo após cada medida 
efetuada. Para lidar com essa variável adicional, sugere-se o uso do método dos Quadrados Latinos.

O método é adequado e deve ser utilizado nos casos em que se suspeita do efeito da ordem sobre as medições. A comple-xidade de cálculo é bem maior que a do método das amplitudes mas é bem semelhante à do método ANOVA. Com a possibilidade de utilizar computadores (pla-nilhas eletrônicas e outros softwares esta-tísticos) toda a complexidade de cálculo desaparece, restando o cuidado na análise dos resultados.

Este trabalho foi todo desenvolvido para operações de medição de torque, porém, durante o desenvolvimento surgiram outras situações em que este modelo foi utilizado com sucesso. Algumas características químicas, por exemplo, alteram-se rapidamente após a retirada das amostras e portanto cada medição se encontra em uma faixa de valores. Para poder "descontar" este efeito e avaliar o R\&R deste sistema de medição, o modelo dos Quadrados Latinos é adequado.

Como parte deste trabalho foi desenvolvida uma planilha eletrônica que efetua os cálculos e imprime os resultados (exemplo no anexo 2).

\section{Anexos:}

\section{ANEXO 1}

O trabalho desenvolvido por JOHNSON (1951) sugere a utilização dos "median-ranks" ou "medianas de ordem" para plotar a função de densidade de probabilidade acumulada $[\mathrm{F}(\mathrm{x})]$ de amostras pequenas $(\mathrm{N}<50)$. Tais “median-ranks" são obtidos em função do tamanho da amostra "N" e da posição "i" de cada elemento, após terem sido ordenados de forma crescente. Exis-tem tabelas para os "median-ranks" mas podese utilizar a fórmula aproximada:

$$
\text { median-rank }(\mathrm{i}, \mathrm{N})=\frac{\mathrm{i}-0,3}{\mathrm{~N}+0,4}
$$

A fórmula produz resultados muito próximos aos valores tabelados, sendo de muito mais fácil utilização.

Comparativo entre median-ranks tabelados e calculados pela fórmula para $\mathrm{N}=10$

\begin{tabular}{|r|c|c|c|c|c|c|c|c|c|c|}
\hline Median-rank & 1 & 2 & 3 & 4 & 5 & 6 & 7 & 8 & 9 & 10 \\
\hline Tabelado & 0,0670 & 0,1632 & 0,2594 & 0,3557 & 0,4519 & 0,5481 & 0,6443 & 0,7406 & 0,8368 & 0,9330 \\
\hline Calculado & 0,0673 & 0,1635 & 0,2596 & 0,3558 & 0,4519 & 0,5481 & 0,6442 & 0,7404 & 0,8365 & 0,9327 \\
\hline
\end{tabular}

Plotando os dados (x) versus os "median-ranks" $[\mathrm{F}(\mathrm{x})]$ podemos analisar o com- portamento dos dados e estimar parâmetros. 


\section{Referências Bibliográficas:}

\section{AUTOMOTIVE INDUSTRY ACTION}

GROUP: Measurement Systems Analysis - Reference Manual. Troy, 1990.

JOHNSON, L.G.: “The median ranks of sample values in their population with an application to certain fatigue studies”. Industrial Mathematics, Vol.2, p.1-9, 1951.
MONTGOMERY, Douglas C.: Design and Analysis of Experiments, $2^{\text {nd }}$ edition. John Wiley \& Sons, Singapore, 1983.

PAULL, A. E.: "On a preliminary test for pooling mean squares in the Analysis of Variance”. Annals of Mathematical Statistics, Vol. 21, s.l, p. 539, 1950.

\section{Bibliografia Complementar:}

COCHRAN, Willian G. \& COX, Gertrude M.: Experimental Designs, ( $2^{\text {nd }}$ edition). John Wiley \& Sons, New York, 1957.

\section{MEASUREMENT SYSTEM ANALYSIS USING LATIN SQUARES METHOD}

\section{Abstract}

Traditional methods used for the evaluation of measurement systems are presented. These traditional methods present deficiencies when the measured characteristic is cumulative (ex.: torque). An alternative method based on Latin Squares is suggested to deal with this type of characteristic. Practical cases, confirming the proposed method's effectiveness, are presented. 